Size of bone flap in decompressive craniectomy: an analysis of complications and prognosis in traumatic brain injury

\title{
Min Xu
}

Nanjing University of Chinese Medicine

Yu Luo

Yangzhou University

Pan Yi

Yangzhou University

Cunzu Wang ( $\nabla$ neurosurgeonwang@126.com )

Yangzhou University https://orcid.org/0000-0001-9012-512X

Research article

Keywords: decompressive craniectomy, size of bone flap, traumatic brain injury, complication, prognosis

Posted Date: October 15th, 2019

DOI: https://doi.org/10.21203/rs.2.13753/v2

License: (c) (i) This work is licensed under a Creative Commons Attribution 4.0 International License. Read Full License 


\section{Abstract}

Abstract Objective: To investigate association of size of bone flap with common complications and prognosis in traumatic brain injury. Methods: A retrospective analysis was performed in $108 \mathrm{TBI}$ patients of Northern Jiangsu People's Hospital from January 2018 to March 2019. Patients' gender, age, Glasgow Coma Scale at admisson, pupils reactivity to the light, size of bone flap, types of craniocerebral injuries and injury locations were recorded. Prognostic indicators including changes in hematoma volume and neurological status were extracted. Statistical methods were conducted to evaluate drug efficacy. Prognostic indicators including Glasgow Outcome Scale scores at discharge and GOS scores of 6 months after operation were extracted to evaluate surgical effcacy. Results : Postoperative complications such as encephalocele and subdural effusion were significantly associated with size of bone flap ( $P$ $<0.05)$. The incidence of encephalocele and SE increased with bone flap size in bone flap groups. Age, GCS at admisson, pupils reactivity to the light, and size of bone flap were shown significantly differences between prognosis groups $(P<0.05)$. By binary logistic regression, Age, pupils reactivity to the light, and the size of bone flap showed statistical significance $(P<0.05)$. Conclusion: Size of bone flap in decompressive craniectomy is a dependent factor to prognosis. Avoiding oversize craniectomy may bring less complications and positive prognosis.

\section{Introduction}

Traumatic brain injury (TBI) is a common neurosurgical disease.The incidence of craniocerebral trauma in China is more than 100 per 10 million, approaching to the level of western developed countries (150200 per 10 million) [1]. Patients with TBI may involve primary injury or develop secondary insult, which can lead to increased intracranial pressure (ICP). Increased ICP is one of the important secondary insults after $\mathrm{TBI}$, and is a main goal of medical and surgical therapy [2]. In severe TBI囚intracranial pressure volume-pressure curve illustrates that when the ICP is decompensated, a small increase in the cranial contents can cause a sharp rise of ICP. On the contrary, a small reduction in the cranial contents may cause signifcant decline of intracranial pressure [3]. A variety of measures have been used in the clinical treatment of elevated ICP.

The primary goal of surgical intervention is the decline of ICP due to eliminating mass effect in severe TBIs [4]. Both craniotomy and craniectomy can serve this purpose. Surgical decompression craniotomy (DC), which still remains controversial, is widely practiced in the control of elevated ICP. It has been advocated in a variety of forms for several decades $[5,6]$. Some studies have recommended the best size of bone flap to effectively manage ICP [7].The best size of bone flap was an independent factor in ICP reduction but not for the overall neurologic outcome [8]. In addition, severe TBIs may still bring multiple complications [9]. In this study, we focused on the size of bone flap of decompressive craniectomy and further investigated its complications and prognosis in traumatic brain injury.

\section{Patients And Methods}




\subsection{Patients collection}

In this study, we did a retrospective analysis. We collected 108 patients with TBI from January 2018 to March 2019 in Northern Jiangsu People's Hospital. Our research had been approved by Institutional Review Board (IRB) and all clinical investigations had been conducted according to the principles expressed in the Declaration of Helsinki. The inclusion standards: all the patients were diagnosed as traumatic brain injuries, performed unilateral or bilateral supratentorial surgeries, and more than 14 years old. Patients with multiple injuries, unstable vital signs and postoperative survival time less than 7 days were excluded.

Patients' gender, age, Glasgow Coma Scale (GCS) at admisson, pupils reactivity to the ligh, size of bone flap, types of craniocerebral injuries (brain contusion, brain hemorrhage, subarachnoid hemorrhage, subdural hemorrhage, and epidural hemorrhage) and injury locations were recorded. Postoperative complications included rebleeding, encephalocele, cerebral infarction, subdural effusion (SE), cerebrospinal fluid leakage from incision, intracranial infection, paradoxical herniation, hydrocephalus , skull defect syndrome and epilepsy. Prognostic indicators including Glasgow Outcome Scale (GOS区 scores at discharge and GOS scores of 6 months after operation were extracted to evaluate surgical effcacy.

\subsection{Bone flap calculation and classification}

Size of bone flap was calculated as $A=\pi \times\left[(d / 2)^{2}+h^{2}\right]$. In which $d$ represents the maximum diameter of the bone window, and $h$ represents the height of the bone window (Maximum distance from the scalp to the maximum diameter of the bone window) (Fig. 1) .

According to the size of bone flap, patients were divided into three groups: the small bone flap group $\left(<110 \mathrm{~cm}^{2}\right)$, the middle bone flap group $\left(110-190 \mathrm{~cm}^{2}\right)$, and the large bone flap group $\left(>190 \mathrm{~cm}^{2}\right)$. According to GOS scores of 6 months after operation, it was divided into good prognosis group (GOS 4-5) and poor prognosis group (GOS 1-3).

\subsection{Statistical Analysis}

All the variables were coded as qualitative or quantitative and analyzed by SPSS19.0 statistical package. A descriptive analysis was performed on the entire patient sample. Nonparametric rank sum test, $T$ test and $\chi 2$ test (with Yates correction when indicated) were used to compare categorical qualitative variables. Data is expressed as Median \pm Quartile and $p$-value of less than 0.05 was considered significant.

\section{Results}

\subsection{Patients}

The eligible 108 patients were $53.7 \pm 15.0$ (14-83) years old; 70, male; 38 female. Most patients had more than one type of craniocerebral injury; brain contusion, 94; brain hemorrhage, 90; subarachnoid 
hemorrhage, 93; subdural hemorrhage, 89; and epidural hemorrhage, 29. Seven patients had one injured region (Frontal, temporal, parietal or occipital lobe), thirty- five patients had two injured regions, thirty- five patients had three injured regions, and thirty- one patients had four injured regions; unilateral injury, 43; bilateral injury, 65; GCS3- 8, 71 (65.7\%); GCS9-15区37 (34.3\%); No pupil dilated, 84 (77.8\%), one pupil dilated, 18 (16.7\%); bilateral pupils dilated, 6 (5.6\%); unilateral DC, 96 (88.9\%); bilateral DC, $12(11.1 \%)$. The average area of the bone window were $123.5 \mathrm{~cm}^{2}\left(34.22-267.1 \mathrm{~cm}^{2}\right)$. Rebleeding $63(58.3 \%)$, encephalocele $52(48.1 \%)$, cerebral infarction 29 (26.9\%), subdural effusion $43(39.8 \%)$, cerebrospinal fluid leakage from incision 2 (1.9\%), intracranial infection 2 (1.9\%), paradoxical herniation $1(0.9 \%)$, hydrocephalus32 (29.6\%), skull defect syndrome57 (52.8\%) and epilepsy $3(2.8 \%)$ were main postoperative complications.

\subsection{Bone flap size and complications}

All 108 patients were divided into three groups, small bone flap group 46 (42.6\%), middle bone flap group 53 (49.1\%), and large bone flap group 9 (8.3\%). The admission GCS was 7.72 \pm 3.24 in group S, 7.38 \pm 3.44 in group $M$, and $6.11 \pm 3.06$ in group L. Patients' gender,age, GCS at admisson, pupils reactivity to the light, size of bone flap, types of craniocerebral injuries and injury locations were all shown non-significantly differences in bone flap groups $(P>0.05)$.

Encephalocele and subdural effusion were shown significant differences among bone flap groups $(P<0.05)$. The incidence of encephalocele was $32.61 \%, 58.49 \%, 66.67 \%$ and incidence of SE was $26.09 \%$, $49.09 \%, 55.56 \%$. The results showed that the incidence of encephalocele and SE increased with bone flap size in three groups. In addition, postoperative complications such as rebleeding, cerebral infarction, cerebrospinal fluid leakage from incision, intracranial infection, paradoxical herniation, hydrocephalus, skull defect syndrome and epilepsy were shown non-significant differences in bone flap groups $(P>0.05)$. In prognostic indicators, GOS scores at discharge were shown non-significant differences $(P>0.05)$ while GOS scores of 6 months were shown significant differences $(P<0.05)$ in bone flap groups (Table 1$)$.

\subsection{Risk factors for prognosis}

108 patients were divided into two groups, good prognosis group 83 (76.85\%) and poor prognosis group 25 (23.15\%). Age, GCS at admisson, pupils reactivity to the light, and size of bone flap were shown significant differences between prognosis groups $(P<0.05)$. While patients' gender,brain contusion, brain hemorrhage, subarachnoid hemorrhage, subdural hemorrhage,epidural hemorrhageand injury locations were all shown non-significant differences between prognosis groups $(P>0.05)$ (Table 2).

Age, GCS at admisson, pupils reactivity to the light, and size of bone flap were further analyzed by binary logistic regression, and the results were shown in Table 3. Age, pupils reactivity to the light, and size of bone flap showed statistical significance $(P<0.05)$. They were dependent factors to GOS scores of 6 months. Patients may got good prognosis with no pupil dilated, young age, or small size of bone flap.

\section{Discussion}


An elevated ICP, which results from primary injury or develop secondary insult, has been proved to be associated with poor outcomes after TBI $[10,11]$. Surgical management is often the treatment option for the TBI patients. By enlarging cranial volume in a short time, DC is a common surgical method for critical patients with uncontrollable ICP. Although DC can reduce mortality in emergency situations, patients may still suffer from poor long-term efficacy $[12,13]$. These results are partly due to pathophysiological changes and high rate of complications secondary to DC. Rebleeding, encephalocele, cerebral infarction, SE, cerebrospinal fluid leakage from incision, intracranial infection, paradoxical herniation, hydrocephalus, skull defect syndrome and epilepsy are main complications [14- 16]. In this study, the incidence of common complications ranged from $0.9 \%$ to $58.3 \%$.

Paradoxical herniation is an unusual complication that tends to occur when there is negative, subatmospheric intracranial pressure under the caved-in scalp flap causing the brain to herniate. Due to the recovery of cerebral perfusion pressure, cerebral blood flow rapidly increases, causing severe congestion and edema of the brain tissue or diffuse brain swelling, which bulges along the defect bone window. Postoperative brain swelling is related to the size of the bone flap. With larger bone flap and greater reduction of ICP, the more the brain flow increases after cerebral perfusion recovery, the more severe the congestion and edema of the brain tissue are, and the higher the incidence of brain swelling is. In addition, some small blood vessels which lose the regulation function are ruputured and bleed to form hematoma with the sharp change of intravascular and extravascular pressure difference [17]. The mechanism is assoiated with decreased ICP after DC.

Sakka [18] believe that the process of absorption of cerebrospinal fluid (CSF) through arachnoid granules is pressure-dependent, that is, the higher the subarachnoid pressure is, the faster the CSF absorption rate is. SE may be formated by the changes in CSF absorption resulting from changes of subarachnoid pressure and ICP. Meanwhile, encephalocele easily "pulls" the contralateral cerebral hemisphere, causing a negative pressure between the contralateral cerebral hemisphere convex surface and the dura mater [19]. Then SE may be formed by the CSF aggregating under the subdural. Encephalocele and subdural effusion were shown significant differences among bone flap groups. The results showed that the incidence of encephalocele and SE increased with bone flap size in three groups.

There are multiple factors which are proved to influence the prognosis TBI, including age, time from injury to treatment, pupillary abnormalities, lesion size, preoperative ICP and GCS score [20, 21]. In this study, age, GCS at admisson, pupils reactivity to the light, and size of bone flap were shown significantlly assoiated with GOS scores of 6 months. In our clinical work, little evidence is available regarding the best practice for the size of bone flap removal. Some studies have suggested that a "large" decompressive craniectomy, no smaller than $12 \times 15 \mathrm{~cm}$ or surface areas of $7000-16,000 \mathrm{~mm}^{2}$, is recommended for an uncontrolled ICP [8, 22]. By binary logistic regression, size of bone flap was still recognized as dependent factors to prognosis. Patients may get good prognosis with a relative smaller size of bone flap. That is to say, a "large" decompressive craniectomy may not bring a positive long-term prognosis. 
In addition, this study has several limitations. The main limitation is the retrospective study design and the reliance on medical records. Data collection and imaging reviews are less complete and accurate than a planned research. And we can not recommend an exact area size to avoid common complications. In spite of these limitations, this study provides useful information to identify size of bone flap in decompressive craniectomy, which can evaluate prognosis and establish therapeutic strategies.

\section{Abbreviations}

TBI: Traumatic brain injury; ICP: Intracranial pressure; DC: Decompression craniotomy; GCS: Glasgow Coma Scale; SE: Dubdural effusion; GOS: Glasgow Outcome Scale; CSF: Cerebrospinal fluid

\section{Declarations}

\section{Acknowledgements}

Not applicable.

\section{Authors' contributions}

All authors have read and approved the manuscript. WCZ and XM contributed to the study conception and design. LY and YP contributed to the acquisition of data. LY was involved in the analysis and interpretation of histological data. The statistical analysis and overall interpretation of data was performed by WCZ, XM and LY. And XM contributed to manuscript drafting.

\section{Funding}

There are no resources of funding to be reported or declared.

\section{Availability of data and materials}

The raw data supporting our findings which were used and/or analyzed during the current study are available in the Archive of Northern Jiangsu People's Hospital. These data can be requested from the corresponding author on reasonable request.

\section{Ethics approval and consent to participate}

Our research had been approved by Institutional Review Board (IRB) of Northern Jiangsu People's Hospital and all clinical investigations had been conducted according to the principles expressed in the Declaration of Helsinki.

\section{Consent for publication}

Not applicable. 


\section{Competing interests}

The authors declare that they have no competing interests.

\section{References}

[1] Zafar SN, Millham FH, Chang Y et al (2011) Chronic subdural hematoma in the elderly: not a benign disease. Journal of Neurosurgery 114(1): 72-6

[2] Jehan F, Azim A, Rhee P et al (2017) Decompressive craniectomy vs. craniotomy only for intracranial hemorrhage evacuation: a propensity matched study. Journal of Trauma and Acute Care Surgery, 83(6):1

[3] Wu R, Shi J, Cao J et al (2017) Two occurrences of delayed epidural hematoma in different areas following decompressive craniectomy for acute subdural hematoma in a single patient: a case report. BMC Surgery, 17(1):123

[4] Talbott J F, Gean A, Yuh EL (2014) Calvarial fracture patterns on CT imaging predict risk of a delayed epidural hematoma following decompressive craniectomy for traumatic brain Injury. Ajnr American Journal of Neuroradiology, 35(10):1930-5

[5] Yoon S Y , Choi YJ , Park SH et al (2017) Traumatic brain injury in children under age 24 months: analysis of demographic data, risk factors, and outcomes of post-traumatic seizure. J Korean Neurosurg Soc, 60(5):584-590

[6] Danish SF , Barone D , Lega BC et al (2009) Quality of life after hemicraniectomy for traumatic brain injury in adults. Neurosurgical FOCUS, 26(6):E2

[7] Carney N , Totten A M , Cindy O'Reilly et al (2016) Guidelines for the Management of Severe Traumatic Brain Injury. Fourth Edition. Neurosurgery, 80(1):1

[8] Reid P, Say I, Shah S, Tolia S et al (2018) Effect of bone flap surface area on outcomes in decompressive hemicraniectomy for traumatic brain injury. World Neurosurg. Nov;119: e922-e927

[9] Moringlane RB, Keric N3, Freimann FB et al (2017) Efficacy and safety of durotomy after decompressive hemicraniectomy in traumatic brain injury. Neurosurg Rev. Oct;40(4): 655-661

[10] Manet R, Schmidt E, François Vassal et al (2016) CSF Lumbar drainage: a Safe surgical option in refractory intracranial hypertension associated with acute posttraumatic external hydrocephalus. Acta Neurochirurgica Supplement, 122(122):55

[11] Ho KM, Honeybul S, Ambati R et al (2018) Prognostic significance of magnetic resonance imaging in patients with severe nonpenetrating traumatic brain injury requiring decompressive craniectomy. World Neurosurg. Apr;112:277-283 
[12] Soustiel J F , Sviri G E , Mahamid E et al (2010) Cerebral blood flow and metabolism following decompressive craniectomy for control of increased intracranial pressure. Neurosurgery, 67(1):65-72

[13] Honeybul S , Ho K (2015) Long-term outcome after decompressive craniectomy for severe traumatic brain injury. Journal of Critical Care, 30(4):851

[14] Dobran M , Mancini F , Nasi D et al (2017) A case of deep infection after instrumentation in dorsal spinal surgery: the management with antibiotics and negative wound pressure without removal of fixation. Bmj Case Rep, Jul 28;2017

[15] Dobran M , Marini A, Gladi M et al (2017) Deep spinal infection in instrumented spinal surgery: diagnostic factors and therapy. G Chir. 38(3):124-129

[16] Dobran M, Marini A, Nasi D et al (2017) Risk factors of surgical site infections in instrumented spine surgery. Surg Neurol Int. Sep 6;8:212

[17] Avecillas-Chasin, Josué M (2015) Subdural effusion in decompressive craniectomy. Acta Neurochirurgica, 157(12):2121-2123

[18] Sakka L , Coll G , Chazal J (2011) Anatomy and physiology of cerebrospinal fluid. Eur Ann Otorhinolaryngol Head Neck Dis, 128(6):309-316

[19] Zhu L , Chu S , Feng D F (2017) Ommaya reservoir implantation for the treatment of contralateral progressive traumatic subdural effusion secondary to decompressive craniectomy: a case report. British Journal of Neurosurgery, Br J Neurosurg. Oct;31(5):628-629

[20] Ecker R D , Mulligan L P , Dirks M et al (2011) Outcomes of 33 patients from the wars in Iraq and Afghanistan undergoing bilateral or bicompartmental craniectomy. Journal of Neurosurgery, 115(1):124129

[21] Van L N , Lingsma H F , Perel P et al (2012) Prognostic value of major extracranial injury in traumatic brain injury: an individual patient data meta-analysis in 39,274 patients. Neurosurgery, 70(4):811

[22] Kochanek PM, Tasker RC, Carney N et al (2019) Guidelines for the management of pediatric severe traumatic brain injury, third edition: update of the brain trauma foundation guidelines, executive summary. Neurosurgery. Jun 1;84(6):1169-1178

\section{Tables}

Table 1. Group differences of complications in three bone flap groups 


\begin{tabular}{|c|c|c|c|c|c|}
\hline & $\underset{(\mathrm{n}=46)}{\operatorname{Group} \mathrm{S}^{\mathrm{a}}}$ & $\begin{array}{c}\text { Group M } \\
(\mathrm{n}=53)\end{array}$ & $\begin{array}{c}\text { Group L } \\
(\mathrm{n}=9)\end{array}$ & Statiscs & $P$ \\
\hline Gender (male:female) & $31 / 15$ & $35 / 18$ & $4 / 5$ & 1.81 & 0.41 \\
\hline Age(range) & $55.65 \pm 14.23$ & $52.04 \pm 16.04$ & $53.67 \pm 13.30$ & 86.91 & 0.69 \\
\hline GCS at admisson & $7.72 \pm 3.24$ & $7.38 \pm 3.44$ & $6.11 \pm 3.06$ & 26.66 & 0.32 \\
\hline $\begin{array}{l}\text { Pupils (normal: } \\
\text { abnormal) }\end{array}$ & $39 / 7$ & $41 / 12$ & $4 / 5$ & 7.30 & 0.12 \\
\hline \multicolumn{6}{|l|}{ Types of injuries: } \\
\hline Brain contusion & 42/46(91.3\%) & 43/53(81.1\%) & 9/9(100\%) & 3.72 & 0.16 \\
\hline $\begin{array}{l}\text { Brain hemorrhage } \\
\text { SAH }\end{array}$ & $\begin{array}{l}40 / 40(8 / .0 \%) \\
38 / 46(82.6 \%)\end{array}$ & $\begin{array}{l}41 / 53(/ / .4 \%) \\
46 / 53(86.8 \%)\end{array}$ & $\begin{array}{l}9 / 9(100 \%) \\
9 / 9(100 \%)\end{array}$ & $\begin{array}{l}3.60 \\
1.94\end{array}$ & $\begin{array}{l}0.17 \\
0.38\end{array}$ \\
\hline Subdural hemorrhage & $36 / 46(78.3 \%)$ & $44 / 53(83.0 \%)$ & $9 / 9(100 \%)$ & 2.48 & 0.29 \\
\hline Epidural hemorrhage & $12 / 46(26.1 \%)$ & $14 / 53(26.4 \%)$ & $3 / 9(33.3 \%)$ & 0.21 & 0.90 \\
\hline $\begin{array}{l}\text { Injury locations } \\
\square 1 / 2 / 3 / 4 \square\end{array}$ & 5/19/10/12 & 2/14/21/16 & $0 / 2 / 4 / 3$ & 7.64 & 0.27 \\
\hline \multicolumn{6}{|l|}{ Complications: } \\
\hline Rebleeding & $22 / 46(47.8 \%)$ & $35 / 53(66.0 \%)$ & 6/9(66.7\%) & 3.64 & 0.16 \\
\hline halocele & $32.6 \%)$ & $31 / 53$ & $6 / 9(66.7 \%)$ & 7.96 & 0.02 \\
\hline Cerebral infarction & $13 / 46(28.3 \%)$ & $13 / 53(24.5 \%)$ & 3/9(33.3\%) & 0.39 & 0.83 \\
\hline SE & $12 / 46(26.1 \%)$ & $26 / 53(49.1 \%)$ & $5 / 9(55.6 \%)$ & 6.44 & 0.04 \\
\hline CSF leakage & $\begin{array}{ll}2 / 40(4.4 \%) \\
2 / 16(140 \%)\end{array}$ & $\begin{array}{l}0 / 53(0 \%) \\
0 / 53(0 \%)\end{array}$ & 0/9(0\%) & $\begin{array}{l}2.15 \\
2.75\end{array}$ & 0.25 \\
\hline $\begin{array}{l}\text { Intracranial infection } \\
\text { Paradoxical herniation }\end{array}$ & $\begin{array}{c}0 / 46(0 \%) \\
0 / 46\end{array}$ & $1 / 53(1.9 \%)$ & $\begin{array}{l}0 / 9(0 \%) \\
0 / 9(0 \%)\end{array}$ & 1.05 & $\begin{array}{l}0.25 \\
059\end{array}$ \\
\hline Hydrocephalus & $12 / 46(26.1 \%)$ & $15 / 53(28.3 \%)$ & $5 / 9(55.6 \%)$ & 3.22 & 0.20 \\
\hline Skull defect syndrome & $21 / 46(45$ & 31/53(5 & $5 / 9(55.6 \%)$ & 1.66 & 0.44 \\
\hline Epilepsy & $1 / 46(2.2$ & $2 / 53(3.8 \%)$ & $0 / 9(0 \%)$ & 0.51 & 0.77 \\
\hline GOS at admission & 11/35 & $23 / 30$ & $5 / 4$ & 8.45 & 0.21 \\
\hline $\begin{array}{l}(1-3):(4-5) \\
\text { GOS ( } 6 \text { months) }\end{array}$ & $6 / 40$ & $14 / 39$ & $5 / 4$ & 51.18 & 0.00 \\
\hline$(1-3):(4-5)$ & & & & & \\
\hline
\end{tabular}

a S: small bone flap group, M: middle bone flap group, L: large bone flap group.

$\mathrm{b}_{1}$ : one injured part, 2: two injured parts, 3: three injured parts, 4: four injured parts.

Table 2. Prognosis factors of decompression craniotomy surgery in TBI 


\begin{tabular}{lcccc}
\hline & Good Prognosis & Poor Prognosis & Statiscs & $P$ \\
\hline Gender (male:female) & $51 / 32$ & $19 / 6$ & 1.785 & 0.182 \\
Age(range) & $52.05 \pm 14.640$ & $59.24 \pm 15.358$ & 0.008 & 0.036 \\
GCS at admisson & $7.99 \pm 3.176$ & $5.52 \pm 3.151$ & 1.250 & 0.001 \\
Pupils (normal: abnormal) & $11 / 72$ & $13 / 12$ & 16.689 & 0.000 \\
Size of bone flap & $115.36 \pm 40.298$ & $150.72 \pm 52.975$ & 2.534 & 0.001 \\
Brain contusion & $73 / 10$ & $21 / 4$ & 0.266 & 0.606 \\
Brain hemorrhage & $69 / 14$ & $21 / 4$ & 0.010 & 0.919 \\
SAH & $71 / 12$ & $22 / 3$ & 0.097 & 0.755 \\
Subdural hemorrhage & $70 / 13$ & $19 / 6$ & 0.921 & 0.337 \\
Epidural hemorrhage & $24 / 59$ & $5 / 20$ & 0.778 & 0.378 \\
Injury locations ${ }^{\mathrm{b}}$ ¿1/2/3/4 & $5 / 27 / 27 / 24$ & $2 / 8 / 8 / 7$ & 0.125 & 0.989 \\
\hline
\end{tabular}

b1: one injured part, 2: two injured parts, 3: three injured parts, 4: four injured parts.

Table 3. Logistic regression of prognosis factors in TBI

\begin{tabular}{lccrl}
\hline & Good Prognosis & Poor Prognosis & Statiscs & $P$ \\
\hline Age(range) & $52.05 \pm 14.640$ & $59.24 \pm 15.358$ & 0.008 & 0.029 \\
GCS at admisson & $7.99 \pm 3.176$ & $5.52 \pm 3.151$ & 1.250 & 0.241 \\
Pupils (normal: abnormal) & $11 / 72$ & $13 / 12$ & 16.689 & 0.008 \\
Size of bone flap & $115.36 \pm 40.298$ & $150.72 \pm 52.975$ & 2.534 & 0.010 \\
\hline
\end{tabular}

\section{Figures}

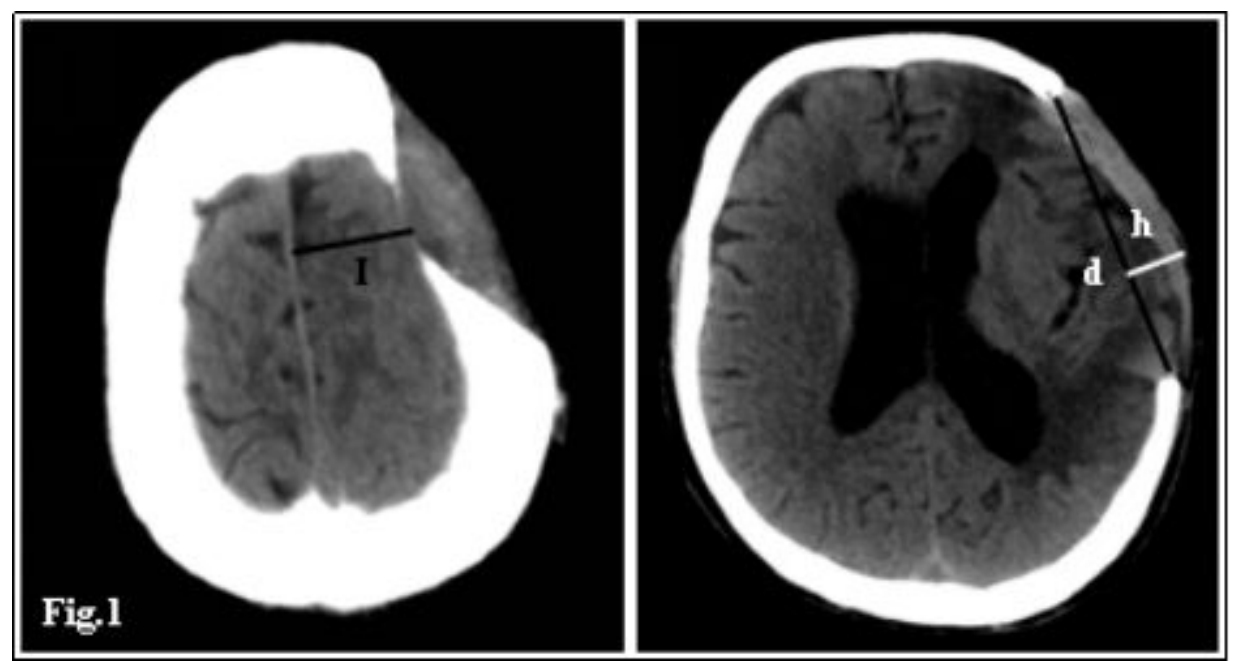

Figure 1

Maximum distance from the scalp to the maximum diameter of the bone window 